\title{
Experiment and Simulation Analysis on Noise Attenuation of Al/MF Cylindrical Shells
}

\author{
Bin Li, ${ }^{1}$ Jian Li, ${ }^{2}$ Shilin Yan, ${ }^{1}$ Wenjie Yan, ${ }^{1,3}$ and $\mathrm{Xu} \mathrm{He}^{1}$ \\ ${ }^{1}$ Hubei Key Laboratory of Theory and Application of Advanced Materials Mechanics, Wuhan University of Technology, \\ Wuhan, Hubei 430070, China \\ ${ }^{2}$ Guangxi Key Laboratory of Automobile Components and Vehicle Technology, Liuzhou, Guangxi 545006, China \\ ${ }^{3}$ Department of Mechanical Engineering, Henan Mechanical and Electrical Vocational College, Xinzheng, Henan 451191, China
}

Correspondence should be addressed to Shilin Yan; yanshl408@126.com

Received 15 June 2017; Accepted 28 September 2017; Published 26 October 2017

Academic Editor: Tai Thai

Copyright (C) 2017 Bin Li et al. This is an open access article distributed under the Creative Commons Attribution License, which permits unrestricted use, distribution, and reproduction in any medium, provided the original work is properly cited.

For the issue concerning internal noise reduction of Al-made cylindrical shell structure, the noise control method of laying melamine foam (MF) layer is adopted for in-shell noise attenuation experiments of $\mathrm{Al}$ and $\mathrm{Al} / \mathrm{MF}$ cylindrical shells and corresponding internal noise response spectrograms are obtained. Based on the Virtual.Lab acoustics software, a finite element model is established for the analysis of noise in the $\mathrm{Al} / \mathrm{MF}$ cylinder shell and numerical simulation computation is conducted for the acoustic mode and in-shell acoustic response; the correctness of the finite element model is verified via comparison with measured data. On this basis, influence rules of different MF laying rate and different laying thickness on acoustic cavity resonance response within the low and medium frequency range of $100-400 \mathrm{~Hz}$ are studied. It is indicated that noise reduction increases with MF laying rate, but the amplification decreases along with the rising of MF laying rate; noise reduction per unit thickness decreases with the increase of laying thickness, while noise reduction per unit area increases.

\section{Introduction}

During the launching process of a carrier rocket, a fairing suffers function of engine jet noise and surrounding aerodynamic noise, which lead to a total sound pressure level (SPL) of $120 \sim 140 \mathrm{~dB}$ in the fairing [1]. In recent years, with the large thrust and high load-oriented development of carrier rocket technologies and application of light, high-strength, and lowdamping composite materials, precise instruments protected by a fairing and other effective loads are under increasingly hostile noise environment. A laying acoustic blanket, one of the most effective measures for noise attenuation in a fairing cavity, has been a focus and difficulty for passive noise control researches and has been extensively studied by researchers both at home and abroad.

Pirk and Souto [2] established a simulation model for an internal laying fiberglass acoustic blanket in the fairing of Brazilian carrier rocket by utilizing statistical energy analysis software, but only discussing noise attenuation effects of sound absorption materials under different thicknesses, densities, and laying rates in medium and high frequency conditions. Gautam [3] laid distributed vibration absorber and a heterogeneous acoustic blanket inside a fairing; the heterogeneous acoustic blanket was made up of heterogeneous mass particle foam materials and showed favorable noise attenuation effect in low frequencies. Similarly, Idrisi [4] embedded multiple mass blocks in foam materials to design heterogeneous blanket and created multiple resonant frequencies by controlling embedding depths of the individual mass blocks, based on which a more extensive low frequency range was achieved with noise attenuation effect. Hughes et al. [5] reduced the noise (frequency range: $200-250 \mathrm{~Hz}$ ) for additional $3 \mathrm{~dB}$ by optimizing density and thickness of cotton fiber and avoided high cost for battery modification. However, both mass and volume increase significantly in the abovementioned methods compared to the original acoustic blanket. Lane et al. [6] conducted an experiment in composite fairing to study denoising effects of the thermal protection system for MF material under three tested bandwidths but rarely involved denoising rules of MF materials in fairing 
under low and medium frequency. There are relatively fewer researches concerning noise attenuation technologies for sound field in a fairing with acoustic blanket. Sun and Pan [7] established a statistical energy model for rocket fairing and suggested that the SPL in an acoustic cavity was highly reduced after laying urea plastic foam in a fairing.

Based on research status concerning noise issue in a fairing in both China and other countries, it can be viewed that porous materials or porous material/other material combinations are mostly adopted for an acoustic blanket. Among common porous materials, MF material shows advantages of lightness, high flexibility, and high acoustical absorption coefficient for medium and high frequencies $[8,9]$ and is applied by US AFRL in design of noise attenuation in composite fairings [6] and is taken by NASA Glenn Research Center as one of the optional noise attenuation technologies for future large carrier rockets [10]. There are a few Chinese researches on in-cavity noise attenuation performance of MF. Shen et al. [11,12] selected MF as their experimental material, set clearance thicknesses in a cavity, filled the clearances, and achieved favorable noise attenuation effects. Liu et al. [13] studied impacts of MF density, porosity, and thickness on cavity noise attenuation performances.

It can be viewed from Chinese and foreign research statuses that there are many studies concerning noise reduction performance of acoustic blanket under high frequency and a rare study involving noise reduction characteristics under low frequency. However, low and medium frequency is a key frequency band for combined application of internal porous material and resonance sound absorber; when the resonant absorption coefficient is close to the maximum value of 0.9 , the combined resonant frequency is not more than $400 \mathrm{~Hz}$ [14]. Therefore, the paper refers to cylindrical section structure of a carrier rocket fairing to construct a scale cylinder finite element model and noise experimental devices. Typical porous material, MF, was adopted as an acoustic blanket laid on internal walls of cylinder shells. According to objective experimental conditions and application range of the simulation software, studying noise reduction rules in MF material cavity under the range of $100-400 \mathrm{~Hz}$ will provide beneficial reference for studying application rules and characteristics of acoustic blanket for passive noise control.

\section{Experimental Research}

In order to obtain internal and external sound pressure response values of a typical cylinder shell, the cylinder shell was placed in the center of a reverberation room. An experimental device as shown in Figure 1 was constructed. For the cylinder cell, the body is made of $1 \mathrm{~mm}$ thickness aluminum sheet, the diameter is $1050.0 \mathrm{~mm}$, the height is $1115.0 \mathrm{~mm}$, the weight is $15.7 \mathrm{~g}$, there are 6 internal transverse reinforcing ribs and 20 longitudinal reinforcing ribs, and the thickness of every reinforcing rib is $1.5 \mathrm{~mm}$. The upper and lower covers are made of medium-density sheet, the thickness is $15.0 \mathrm{~mm}$, and the average sound insulation volume is $20 \mathrm{~dB}$. As the volume of the reverberation room is small, a rubber band or soft spring-hoisting structure could not be simply

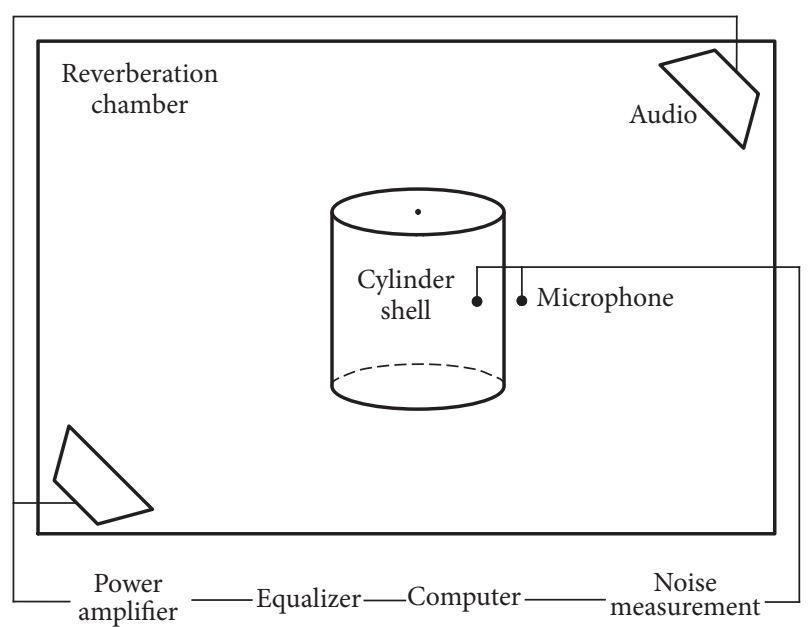

FIGURE 1: Sketch of the noise experiment.

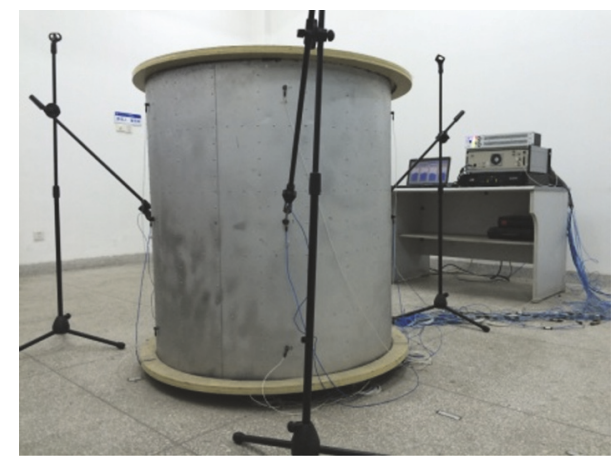

FIGURE 2: Sketch of external sound field test.

applied, and four pieces of $50 \mathrm{~mm}$ thickness shock-reducing rubber blocks were adopted between the lower cover plate of the cylinder shell and the floor to simulate the free state of a cylinder shell.

During practical measurements, Prosig noise and vibration measurement was adopted for objective noise test of the cylinder shell. When setting acoustic microphones inside and outside the cylinder shell, as shown in Figure 2, a total of 3 acoustic microphones were uniformly distributed at $30 \mathrm{~mm}$ sites of extracavity middle layer from the external wall; external sound pressure values were measured; consistency of external load was guaranteed. As shown in Figure 3, incavity area was divided into four planes with 90 degrees apart in radial direction, and every plane was divided into upper, middle, and lower layers; one acoustic microphone was set at the radial center and radial endpoints of every layer; internal sound pressure values were measured against studied rule of in-cavity sound pressure response under different conditions. In conclusion, a total of 3 acoustic microphones and 24 acoustic microphones were set respectively outside and inside the cylinder shell cavity. During the measurement, the signal sampling frequency was $32,000 \mathrm{~Hz}$ and the sampling time was set as $8 \mathrm{~s}$; the uniformity scope of indoor noise load was $\pm 3 \mathrm{~dB}$, and the deviation range of the noise control spectrum was $\pm 5 \mathrm{~dB}$. 


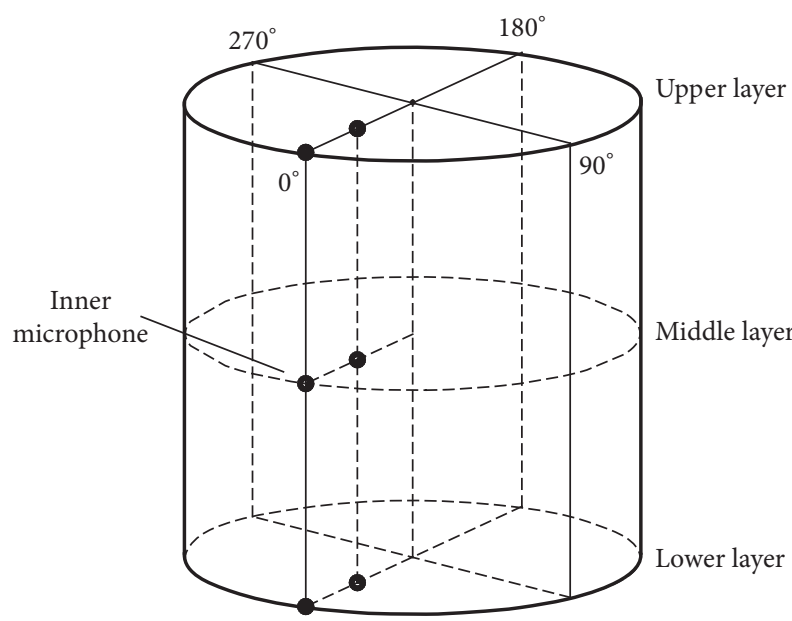

FIGURE 3: Sketch of arrangement of in-cavity acoustic microphones.

\section{Numerical Simulation Analysis}

3.1. Simulation Model. A finite element model for an entire cylinder shell was established on the basis of Hypermesh finite element software; for the model, the cylinder shell and reinforcing rib structure were simulated via CQUAD4 surface element; the acoustic cavity and the upper and lower cover plates were simulated via CHEXA volume meshing; the connection between the reinforcing ribs and the walls was simulated via RBE2 element; and MF was simulated via CHEXA volume meshing with node merging with the internal acoustic cavity mesh.

The established finite element model was introduced in the Virtual.Lab Acoustic simulation software for acoustic meshing pretreatment, which defined the structures, fluid, and material properties, while the cylindrical shell and the reinforcing rib material are aluminum, whose density is $2700 \mathrm{~kg} / \mathrm{m}^{3}$, Young's modulus is $70 \mathrm{GPa}$, and Poisson's ratio is 0.33 . The upper and lower covers are treated as isotropic materials, whose density is $800 \mathrm{~kg} / \mathrm{m}^{3}$, Young's modulus is $2 \mathrm{Gpa}$, and Poisson's ratio is 0.4 . The MF acoustic parameters use the test results from Italian SCS Institute, as shown in Table 1. Meanwhile, established MPC wire jointing elements between the upper and lower cover plates with the shell defined coupling between internal and external cavity acoustic elements and structural elements (including coupling between the internal acoustic cavity with MF and internal surfaces of the upper and lower cover plates and between the external acoustic cavity with the shell and external surfaces of the upper and lower cover plates), and set different tolerances according to clearances existing on coupling surfaces during modeling. Meanwhile, the plane where the lower surface of the external acoustic cavity was located was defined as the reflecting surface to simulate the floor, and the remaining external surfaces were for free ins and outs of sound waves and were defined as automatch layer (AML). See Figure 4 for details.

With the simulation software, distributed plane waves were adopted to stimulate reverberation room environment;

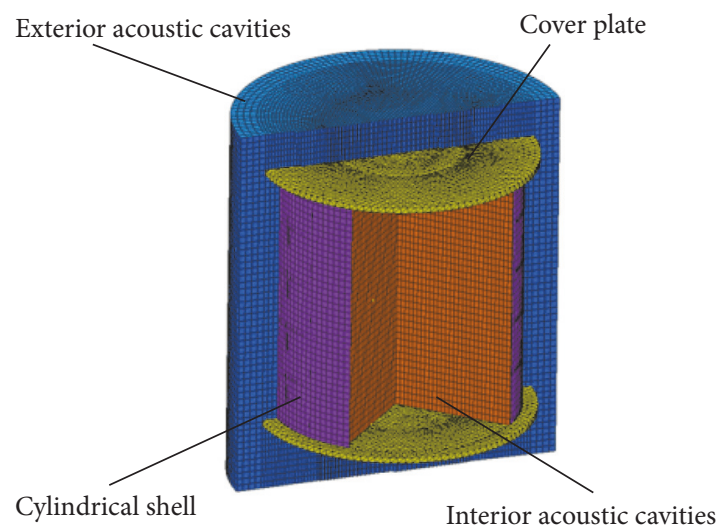

Figure 4: Finite element model map.

TABLE 1: Acoustic parameters of MF.

\begin{tabular}{lc}
\hline Acoustic parameters & Value \\
\hline Flow resistivity $/\left(\mathrm{Pa} \cdot \mathrm{s} \cdot \mathrm{m}^{-2}\right)$ & 10925 \\
Sound speed $/ \mathrm{m} \cdot \mathrm{s}^{-1}$ & 346 \\
Air density $/ \mathrm{kg} \cdot \mathrm{m}^{-3}$ & 1.185 \\
Porosity & 0.99 \\
Tortuosity & 1.02 \\
Viscous characteristic length $/ \mathrm{mm}$ & 0.1 \\
Thermal characteristic length $/ \mathrm{mm}$ & 0.13 \\
\hline
\end{tabular}

the power spectrum inputs of external loads set in the software were actual parameters obtained via experimental measurement; the polarization amount was set as 2 ; the reverberation sound source was evenly divided into 24 plane waves, which were uniformly distributed around the simulation model in a circular shape; superposition of several plane waves formed a reverberation filed; direct sound and vibration coupling calculation was then conducted; according to randomized posttreatment solvers, the random frequency response of the acoustic cavity was analyzed. The range of output frequency was $0-400 \mathrm{~Hz}$, and the step length was $10 \mathrm{~Hz}$; the output form was power spectral density function; the SPL curves were achieved at simulation filed points which were located at same positions of the experimental measurement sites, and rules of sound pressure response were studied at corresponding measurement sites.

3.2. Simulation Results and Comparative Analysis. On the basis of the established simulation model, the correctness of the finite element modeling and simulation computation was verified from three aspects, acoustic mode, cavity acoustic response, and in-cavity acoustic response of laying $\mathrm{MF}$ material.

The mode of cylinder cavity determines internal sound field distribution. According to the analytical formula for intrinsic frequency of a cylinder sound cavity, the theoretical 
TABLE 2: Comparison of acoustic modes achieved by analytical method and simulation computation.

\begin{tabular}{lccccccccc}
\hline Mode & $/ l, m, n$ & $(1,0,0)$ & $(0,1,0)$ & $(1,1,0)$ & $(2,0,0)$ & $(0,2,0)$ & $(1,2,0)$ & $(2,1,0)$ & $(0,0,1)$ \\
\hline Analytical value & $/ \mathrm{Hz}$ & 151.1 & 192.2 & 244.5 & 302.2 & 317.9 & 352.1 & 358.2 & 398.0 \\
Simulation value & $/ \mathrm{Hz}$ & 151.1 & 191.3 & 243.8 & 302.2 & 317.2 & 351.4 & 357.7 & 398.1 \\
Error & $/ \%$ & 0.00 & 0.51 & 0.31 & 0.00 & 0.23 & 0.19 & 0.15 & 0.01 \\
\hline
\end{tabular}

value of the acoustic mode in the cylinder shell is calculated, specifically and it is shown as

$$
f_{l m n}=\frac{c}{2 \pi} \sqrt{\left(\frac{l}{L} \pi\right)^{2}+k_{m n}^{2}} .
$$

In the formula, $c$ refers to sound velocity, $l$ refers to axial mode, $m$ refers to circumferential mode, $n$ refers to radial mode, $L$ refers to the axial length of the cylinder acoustic cavity, $k_{m n}$ represents the number $n$ solution of $j_{m}^{\prime}\left(k_{m n} r\right)=0, j_{m}$ refers to $m$ order of Bessel function, and $r$ refers to radius of the cylinder acoustic cavity.

The results of the analytical values are compared with simulation values, as shown in Table 2. It can be viewed from the following table that the maximal error of analytical results is $0.51 \%$, verifying the correctness of the finite element modeling.

Due to limitation of experimental objective conditions, the measurement values obtained from the 24 acoustic microphones in the cylinder shell show no entire consistency. Therefore, the comparison is conducted globally between simulation values and experimental values of internal acoustic response before and after laying MF. The comparison of internal acoustic sound pressure response averages obtained via numerical computation and experimental measurement before and after laying the material is shown in Figure 5.

It can be viewed from the figure that the experimental results are basically identical with simulation computation results within the frequency range of $100-400 \mathrm{~Hz}$, showing the correctness of the finite element method for the acoustic response computation within low-mid frequency range. Meanwhile, the curve within the range of $0-100 \mathrm{~Hz}$ shows several peaks; it can be known by comparing with structural mode that the fact is caused by structural resonance stimulated by external sound filed; as shown in Figure 5, the peak response frequency near a modality shows left shift after laying material. For example, the peak response frequency near the first-order axial mode $(1,0,0)$ after laying MF material shifts to the left; the difference of response peaks near the first-order axial modal frequency before and after laying the material is caused by higher wave velocity of sound wave in the air than in MF. After laying MF and in order to guarantee the continuity at the contact surface between the acoustic cavity and MF, the axial wave number of the sound wave in the two shall maintain consistency, which leads to reduction of the axial modal frequency of the cavity and further verifies the correctness of the simulation model.

\section{Results and Discussion}

To study noise reduction rules of MF acoustic blanket, the ratio of MF laying area to inner wall area of cylindrical structure is defined as laying rate, and the ratio of MF laying mass to total structural mass is defined as specific gravity ratio. Within the frequency range of $100-400 \mathrm{~Hz}$, numerical computation and analysis are conducted for the 5 resonant frequency orders, $(1,0,0),(0,1,0),(1,1,0),(0,2,0)$, and $(2,1,0)$ in the cylindrical acoustic cavity (Figure 5$)$ to study influence rules of different laying rates, laying thicknesses, and laying areas on acoustic resonance response and to provide beneficial reference for in-depth study on application rules and characteristics of acoustic blanket for passive noise control.

4.1. Impacts of Laying Rate. To explore influence rules of laying rate on noise reduction performance of MF acoustic blanket, numerical computation and analysis of noise reduction rules are conducted for $40 \mathrm{~mm}$ MF with five different laying rates $(0 \%, 25 \%, 50 \%, 75 \%$, and $100 \%)$.

Figure 6 shows average SPL response spectrum of 12 measurement points in a cylindrical acoustic cavity under stimulation of external noise. It can be viewed from the figure that, under an empty-cavity state, resonance points of different orders are stimulated to different extents; due to sound and vibration coupling effect, frequencies of different orders in the acoustic cavities are enhanced to different degrees; within the frequency range of $100-150 \mathrm{~Hz}$, acoustic response of internal cylindrical cavity is determined by structural stiffness; additionally, within the range, response of internal acoustic cavity changes with the variation of noise reduction; the noise reduction is higher and internal acoustic response is higher with a higher laying rate; therefore, internal response of the empty cavity is lowest, while that of the acoustic cavity with full laying ranks at the top [15]. Within the frequency range of $150-400 \mathrm{~Hz}$, the internal SPL curvilinear trends after laying MF materials are basically consistent; a higher laying rate results in better noise reduction outcome; however, when the laying rate is higher than $25 \%$, the overlap ratio of internal SPL curves increases, indicating that the amplification of noise reduction within the frequency range decreases with the increase of MF laying rate.

It can also be viewed from Figure 6 that there are several obvious resonance orders within the frequency range of $100-400 \mathrm{~Hz}$; among those, the response of the $(1,1,0)$ order acoustic cavity resonance is not prominent, which is majorly related to the higher thickness of upper and lower covers than side wall; when MF acoustic blanket is applied, the order resonance is difficult to be identified. For the five resonance orders 


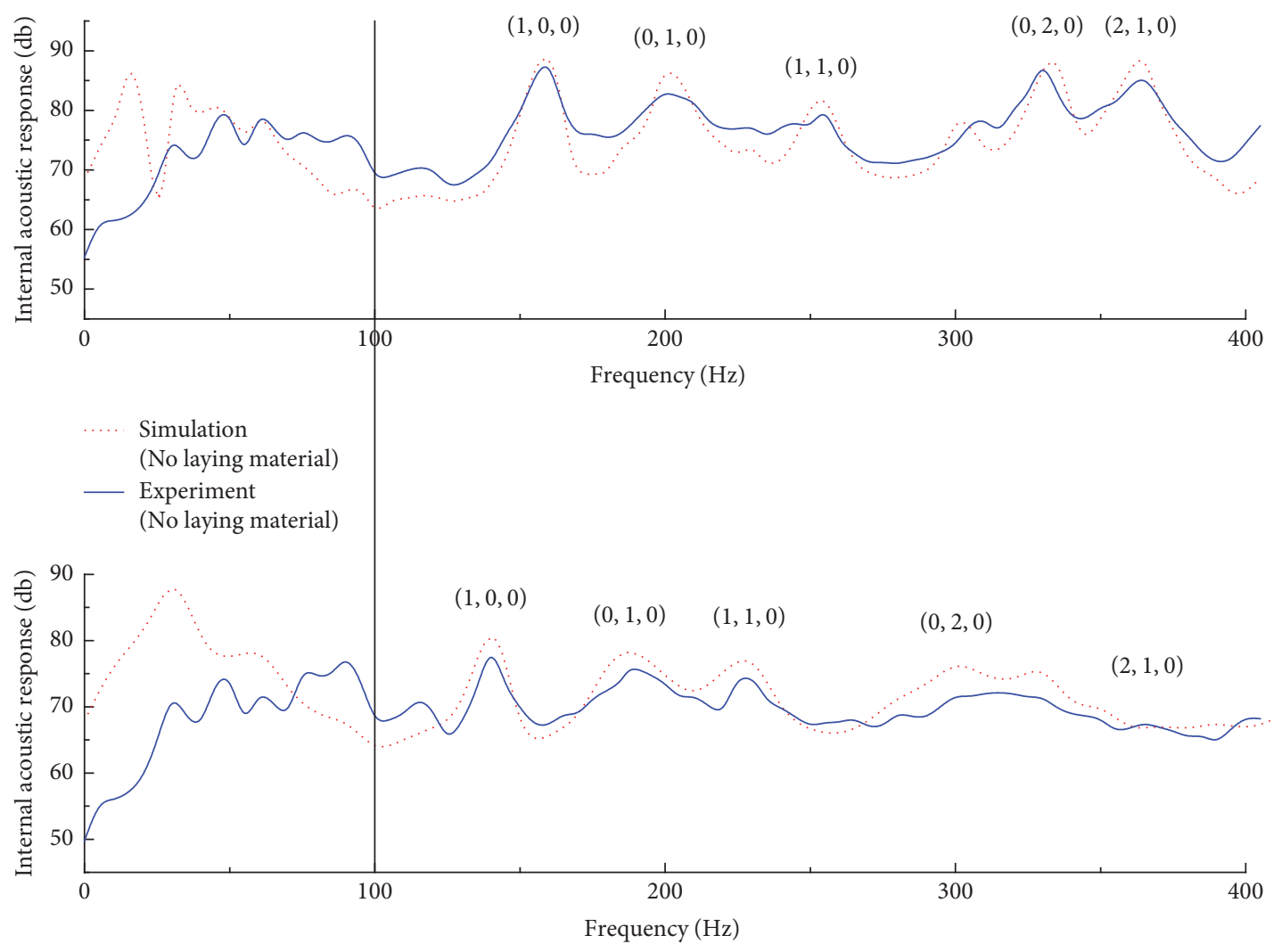

Simulation (laying $40 \mathrm{~mm}$ melamine material)

— Experiment (laying $40 \mathrm{~mm}$ melamine material)

FIGURE 5: Comparison of internal response spectrum achieved from experiment and simulation.

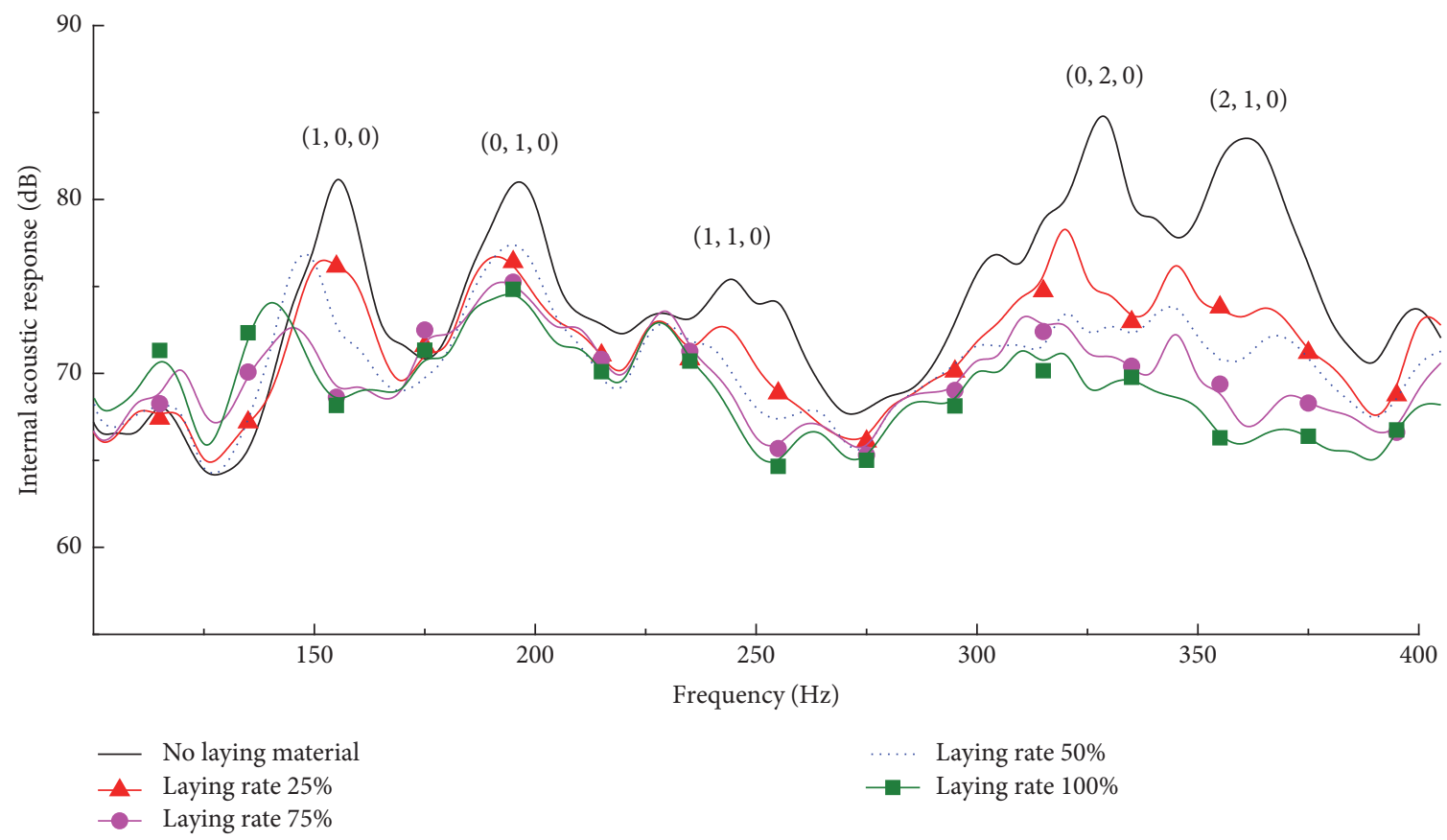

FIGURE 6: Average sound pressure level inside cylinder with different area coverage. 
indicated in Figure 6, acoustic cavity resonance decreases with the increase of material laying rate, suggesting that MF acoustic blanket shows significant denoising performance; it can also be viewed that $75 \%$ and $100 \%$ laying rates show relatively small difference of denoising performance, but better performances than $25 \%$ and $50 \%$ laying rate. With the increase of frequency, medium-high frequency acoustical absorption coefficient of MF increases, and a higher laying rate leads to better noise reduction performance. In addition, for high-order acoustic cavity resonance, the noise reductions with the increase of laying rate are higher than low-order resonance.

To further explore the influence rules of laying rate and specific gravity ratio on noise reduction performance of MF acoustic blanket, numerical analysis and discussion are conducted for noise reductions of $40 \mathrm{~mm}$ MF material in resonance frequency orders of $(1,0,0),(0,1,0),(1,1,0)$, $(0,2,0)$, and $(2,1,0)$ with the frequency range of $100-400 \mathrm{~Hz}$. Noise transmission and acoustic blanket performance were measured by computing the noise reduction, which is defined here to be the ratio of the spatially averaged external sound field impinging on the cylindrical shell to the spatially averaged interior acoustic response. The computation is as follows:

$$
\mathrm{NR}(\mathrm{dB})=20 \log _{10}\left(\frac{\text { External rms }}{\text { Internal } \mathrm{rms}}\right) .
$$

To estimate the "External rms" (where rms denotes rootmean square) of the external sound field, 3 microphones measurements were taken at different locations around the cylindrical cavity exterior and spatially averaged. The "Internal rms" was estimated in a similar way by taking 24 microphones measurements at many locations throughout the cylindrical cavity interior.

In Figures 7 and 8, the noise reduction is presented both as a function of laying rate and as a function of specific gravity ratio. That is to show that curves of noise reduction change with laying rate and specific gravity ratio of $20 \mathrm{~mm}$ and $40 \mathrm{~mm}$ MF materials. Values of the points in the curves are obtained via formula (2). For each order, linear regressions were computed for the corresponding data and are superimposed on the data points. In each case, there were similar trends and the noise reduction appears to be somewhat linear with respect to the amount of acoustic treatment. The midpoint of the curve is the average noise reduction, while the slope of the curve indicates influencing degree. It can be viewed from Figures 7 and 8 that, for $(1,0,0)-,(0,1,0)-,(1,1,0)-,(0,2,0)-$, and $(2,1,0)$-order center frequency points, the average noise reductions are $3.95 \mathrm{~dB}, 1.52 \mathrm{~dB}, 2.33 \mathrm{~dB}, 4.55 \mathrm{~dB}$, and $4.89 \mathrm{~dB}$, respectively, under a specific gravity ratio of $2.25 \%$, and $4.94 \mathrm{~dB}, 2.61 \mathrm{~dB}$, $3.36 \mathrm{~dB}, 5.72 \mathrm{~dB}$, and $6.35 \mathrm{~dB}$ under a specific gravity of $4.50 \%$. It can be viewed that increasing MF thickness significantly increases low and medium frequency sound absorption effects. However, the increase of noise reduction does not show an exact direct proportional relation with the increase of specific gravity ration. Except for the $(1,0,0)$ order, the average noise reduction and the slope of curve of central frequency points both increase with frequency, suggesting

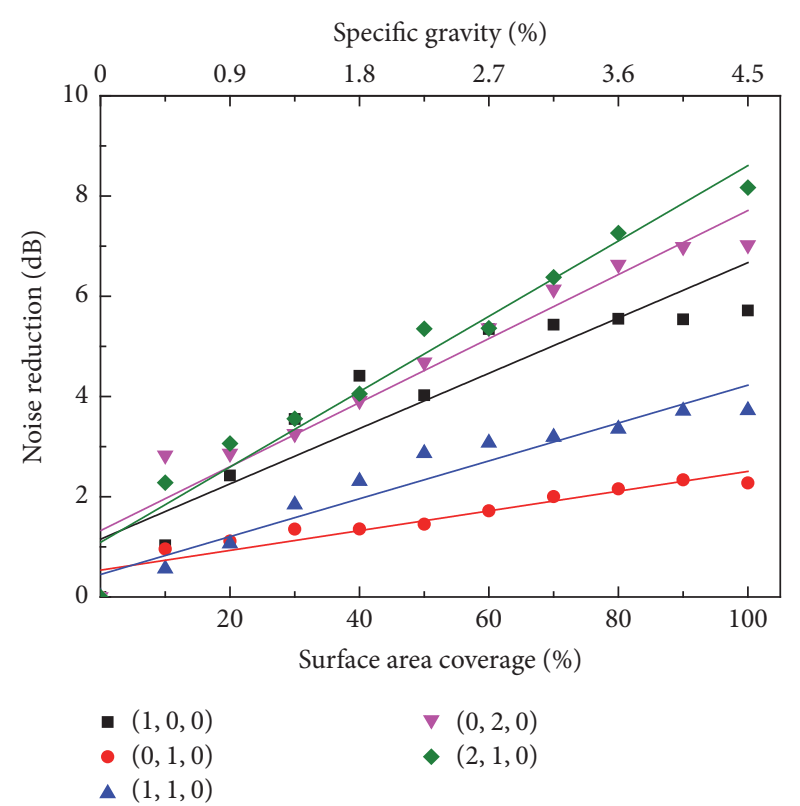

FIGURE 7: Curves of noise reduction with changing of laying rate and proportion $(20 \mathrm{~mm})$.

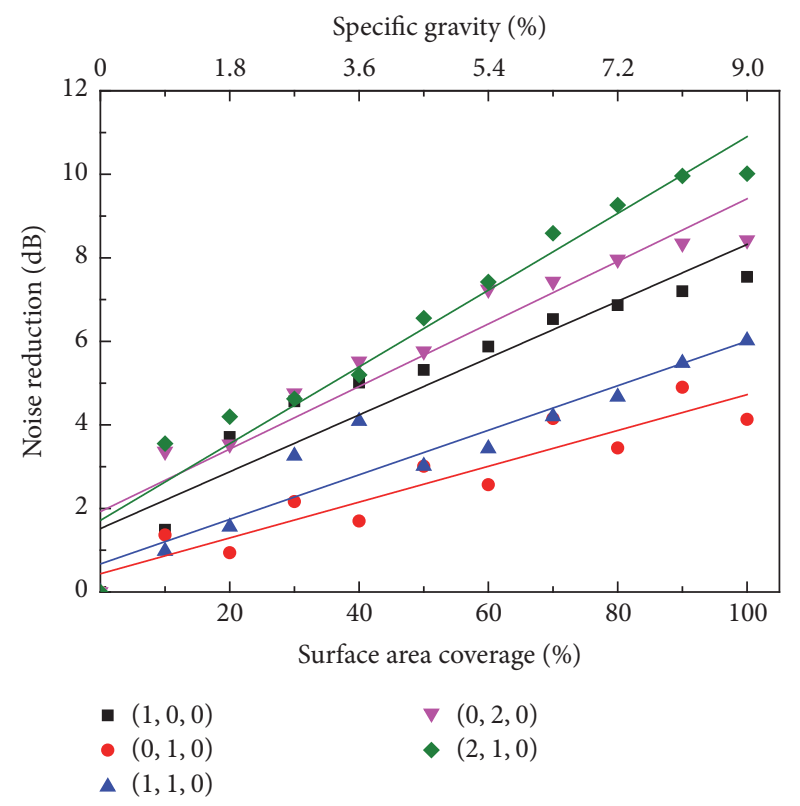

FIGURE 8: Curves of noise reduction with changing of laying rate and proportion $(40 \mathrm{~mm})$.

that noise reduction performance of MF material gradually improves with the increase of frequency and laying rate and that laying rate poses significantly high impacts on noise reduction of $(1,0,0)$-order central frequency point. When the low frequency acoustical absorption coefficient of MF is relatively low, improving laying rate effectively reduces low frequency noise.

4.2. Noise Reductions per Unit Thickness and Unit Area. In addition to satisfying requirements of noise reduction 
TABLE 3: Noise reductions as a function of thickness and surface area coverage.

\begin{tabular}{|c|c|c|c|c|c|c|}
\hline \multirow{2}{*}{ Order } & \multicolumn{3}{|c|}{ Noise reductions per unit thickness $(\mathrm{dB} / 10 \mathrm{~mm})$} & \multicolumn{3}{|c|}{ Noise reductions per unit area $\left(\mathrm{dB} / \mathrm{m}^{2}\right)$} \\
\hline & $20 \mathrm{~mm}$ & $60 \mathrm{~mm}$ & $100 \mathrm{~mm}$ & $20 \mathrm{~mm}$ & $60 \mathrm{~mm}$ & $100 \mathrm{~mm}$ \\
\hline$(1,0,0)$ & 3.62 & 1.48 & 1.03 & 1.95 & 2.39 & 2.70 \\
\hline$(0,1,0)$ & 3.49 & 2.37 & 1.75 & 1.88 & 3.83 & 4.19 \\
\hline$(1,1,0)$ & 5.51 & 3.02 & 2.24 & 2.97 & 4.30 & 5.02 \\
\hline$(0,2,0)$ & 3.13 & 2.94 & 2.02 & 2.69 & 4.01 & 4.34 \\
\hline$(2,1,0)$ & 2.38 & 2.36 & 1.76 & 2.04 & 3.38 & 3.97 \\
\hline SPL & 3.96 & 1.75 & 1.18 & 2.46 & 2.83 & 3.19 \\
\hline
\end{tabular}

performance, structural lightness shall also be considered for realization of engineering application. Therefore, with MF as the study subject, numerical computation is used to study noise reduction characteristics per unit thickness and unit area of MF acoustic blanket.

The total area of internal cylindrical shell wall is $3.709 \mathrm{~m}^{2}$; the noise reductions per unit thickness and unit area are shown as in Table 2. For cases with same laying thickness, maximal noise reductions per unit thickness and unit area are both shown in $(1,1,0)$. It can be viewed from the table that, with the increase of thickness, the noise reduction per unit thickness shows a gradually declining tendency, while the noise reductions per unit area gradually increase with thickness.

It can also be viewed from SPL in Table 3 that the thinner treatment had the higher reduction per unit thickness relative to the other two treatments, which is reasonable since $20 \mathrm{~mm}$ MF covers more surface area than the same amount of the other two groups. For a given mass limit, the $20 \mathrm{~mm}$ foam offered the highest noise reduction per unit thickness which is $3.96 \mathrm{~dB}$. In fact, the $20 \mathrm{~mm}$ material offered nearly 2 to 3 times the performance as the other two groups. The data also indicate that if the surface area available for treatment is limited, but the thickness of the treatment is not, then the $100 \mathrm{~mm}$ treatment offers the best noise control solution, and the noise reduction per unit area for $100 \mathrm{~mm} \mathrm{MF}$ is $3.19 \mathrm{~dB}$. This is reasonable considering that $1 \mathrm{~m}^{2}$ of $100 \mathrm{~mm}$ foam is a considerable amount of acoustic treatment. Therefore, for a given mass limit, smaller laying thickness leads to higher noise reduction per unit thickness. When the laying area is limited, higher laying thickness leads to higher noise reduction per unit area; however, with the increase of laying material thickness, the amplification of noise reduction per unit area gradually decreases.

\section{Conclusion}

In the paper, the noise control method of laying acoustic blanket is adopted to establish a finite element model of cylinder shell and noise experiment device to test internal noise responses of structural cavity of the cylinder shell and cavity with laying MF material. The results obtained via finite element method and experimental method are compared to verify the correctness of the finite element modeling. Based on the finite element model, within the frequency range of $100-400 \mathrm{~Hz}$, numerical computation and analysis are conducted for cylindrical acoustic cavity $(1,0,0),(0,1,0)$, $(1,1,0),(0,2,0)$, and $(2,1,0)$ orders to study impacts of different laying rate, laying thickness, and laying areas on acoustic cavity resonance response. The following conclusions are made:

(1) Within the frequency range of $100-400 \mathrm{~Hz}$, noise reduction increases with the $\mathrm{MF}$ laying rate, but the amplification decreases. In addition, within the frequency range of $100-150 \mathrm{~Hz}$, a higher MF laying rate leads to a higher internal acoustic response; for the frequency range of $150-400 \mathrm{~Hz}$, the internal acoustic response decreases with the increase of MF laying rate.

(2) Within the frequency range of $100-400 \mathrm{~Hz}$, noise reduction performance of $\mathrm{MF}$ material gradually increases along with frequency and laying rate; noise reduction performance improves faster with thicker MF materials. In addition, the impact of laying rate is relatively significant for $(1,0,0)$-order acoustic cavity resonance, and the average noise reductions are $3.95 \mathrm{~dB}(20 \mathrm{~mm} \mathrm{MF})$ and $4.94 \mathrm{~dB}$ (40 $\mathrm{mm} \mathrm{MF})$, respectively.

(3) For a given mass limit, a smaller laying material thickness leads to a higher noise reduction per unit thickness. When the area of laying material is limited, a larger laying material thickness leads to higher noise reduction per unit area; however, with the increase of laying thickness, the amplification of noise reduction per unit area decreases gradually.

\section{Conflicts of Interest}

The authors declare that there are no conflicts of interest regarding the publication of this article.

\section{Acknowledgments}

This work is supported by Guangxi Natural Science Foundation (2016GXNSFAA380211) and Fundamental Research Funds for the Central Universities (WUT: 2017IB016). This work was finished at Wuhan University of Technology (WUT), Wuhan. 


\section{References}

[1] J.-L. Rong, X.-Y. Chen, B. Li, X.-Y. Cheng, and H.-B. Li, "A method for noise attenuation in cylindrical cavity with melamine foam lining," Journal of Astronautics, vol. 37, no. 10, pp. 1271-1278, 2016.

[2] R. Pirk and C. D. Souto, "Implementation of acoustic materials to the VLS-1 fairing - A sensitivity analysis using SEA," Journal of the Brazilian Society of Mechanical Sciences and Engineering, vol. 34, no. 1, pp. 82-88, 2012.

[3] A. Gautam, Design and Development of Advanced Vibration and Noise Control Devices Using Finite Element Analysis, Virginia Polytechnic Institute and State University, Blacksburg, VA, USA, 2005.

[4] K. Idrisi, Heterogeneous (HG) Blankets for Improved Aircraft Interior Noise Reduction, Virginia Polytechnic Institute and State University, Blacksburg, VA, USA, 2005.

[5] W. O. Hughes, A. M. McNelis, and H. Himelblau, "Investigation of acoustic fields for the cassini spacecraft: Reverberant versus launch environments," in Proceedings of the Aeroacoustics Conference and Exhibit, AIAA/CEAS 1999, pp. 1193-1203, Bellevue, Washington, USA, 1999.

[6] S. A. Lane, S. Kennedy, and R. Richard, "Noise transmission studies of an advanced grid-stiffened composite fairing," Journal of Spacecraft and Rockets, vol. 44, no. 5, pp. 1131-1139, 2007.

[7] M. Sun and Z. W. Pan, "Noise Environment Prediction and Anti-acoustic Design of Payload Fairing," Missiles and Space Vehicles, vol. no. 4, pp. 6-10, 2008.

[8] G. H. Yuan, X. C. Wang, P. Z. Hou, and C. L. Li, "Absorbing Property of Open-cell Melamine Foam," International Journal of Mechanical and Materials Engineering, vol. 31, no. 9, pp. 5557, 2007.

[9] J. P. Arenas and M. J. Crocker, "Recent trends in porous soundabsorbing materials," Sound and Vibration, vol. 44, no. 7, pp. $12-$ $17,2010$.

[10] W. O. Hughes, A. M. Mcnelis, and M. E. McNelis, "Acoustic Test Characterization of Melamine Foam for Usage in NASAs Payload Fairing Acoustic Attenuation Systems," in Proceedings of the in Proceedings of the 28th Aerospace Testing Seminar Sponsored by the Aerospace Corporation, Los Angeles, Calif, USA, 2014.

[11] Y. P. Shen, Application of The New Sound-Absorbing Material in Ship Cabin Noise Reduction, Ocean University of China, Qingdao, 2014.

[12] Y. P. Shen, Y. C. Yang, C. L. Chen, and C. Wang, "The effect of the space between trim panel and bulkhead plate on noise reduction of cabin," Ship Ocean Engineering, vol. 43, no. 3, pp. 37-40, 2014.

[13] K. Liu, W. G. Wu, and B. Qiu, "Full spectrum simulation prediction of high-speed vessel cabin noise," Transportation Science and Technology, vol. 2, pp. 109-112, 2010.

[14] L. He, H. C. Zhu, X. J. Qiu, and G. H. Du, Acoustic Theory and Engineering Application, Science Press, Beijing, 2006.

[15] D. Li, Vibro-Acoustic Behavior and Noise Control Studies of Advanced Composite Structures, University of Pittsburgh, Pittsburgh, PA, USA, 2003. 


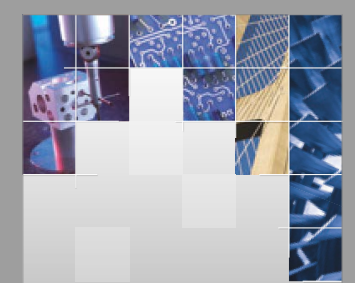

\section{Enfincering}
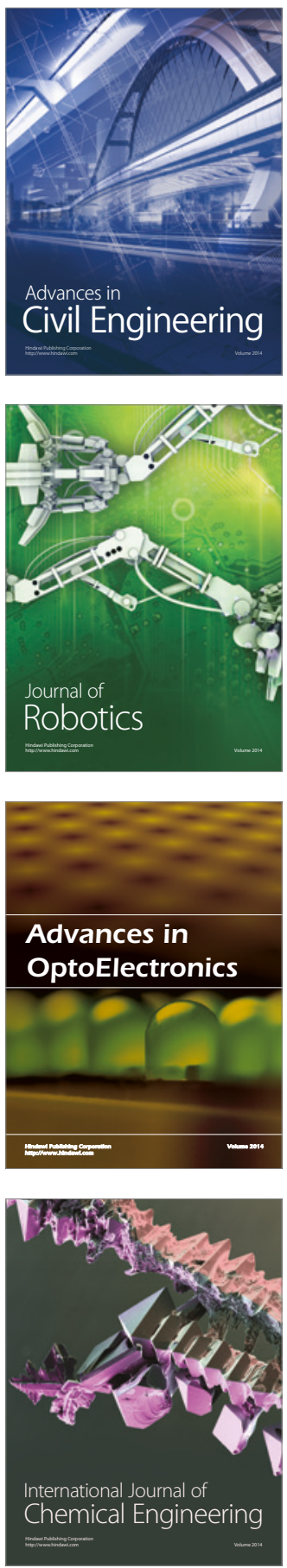

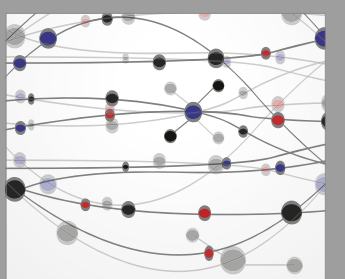

The Scientific World Journal

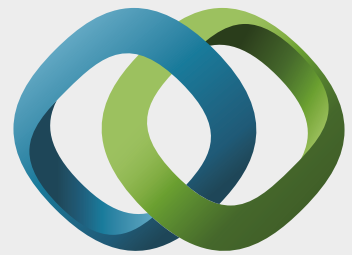

\section{Hindawi}

Submit your manuscripts at

https://www.hindawi.com
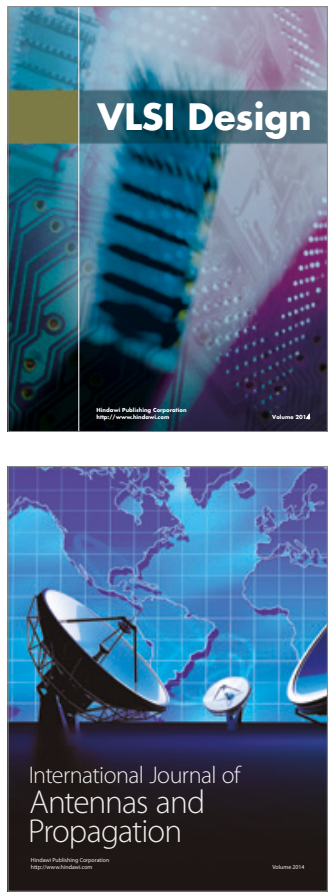

\section{Rotating}

Machinery
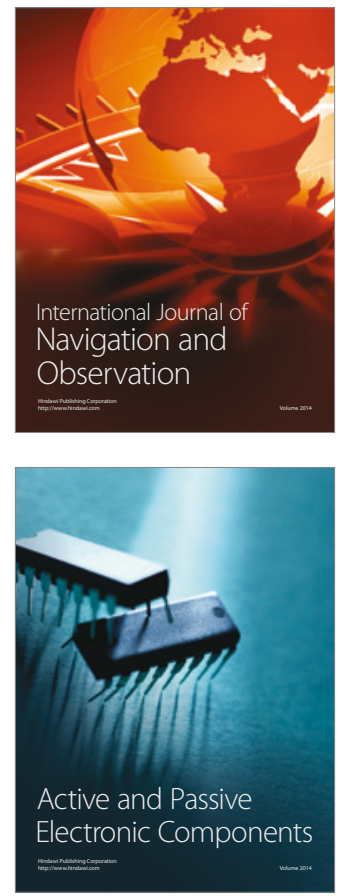
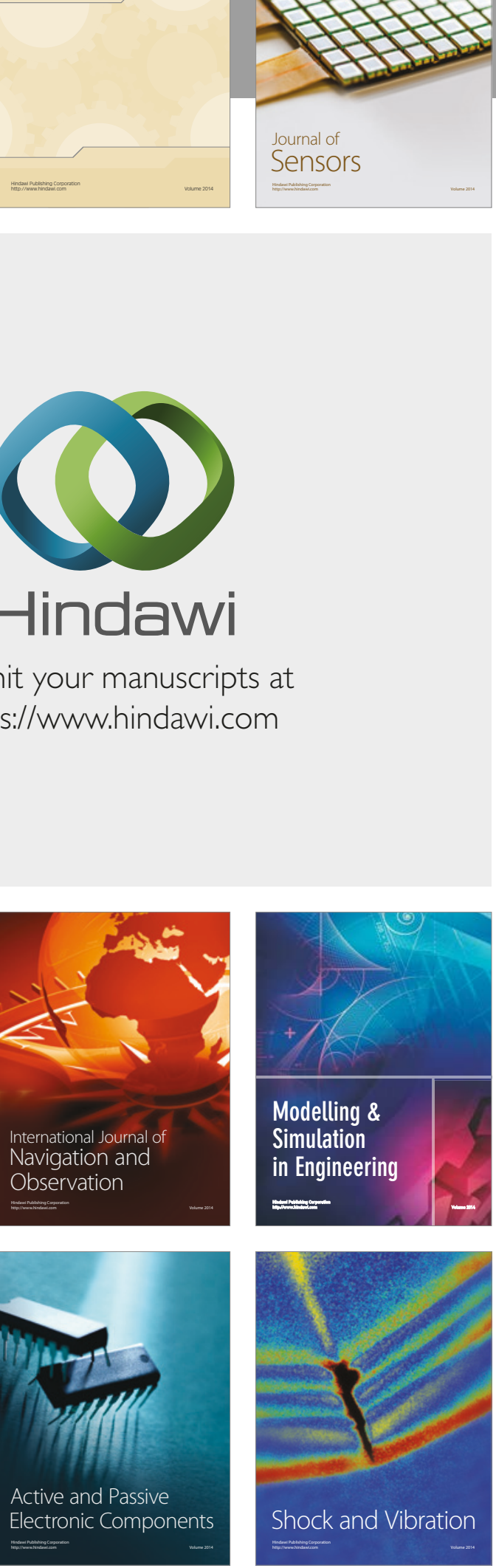
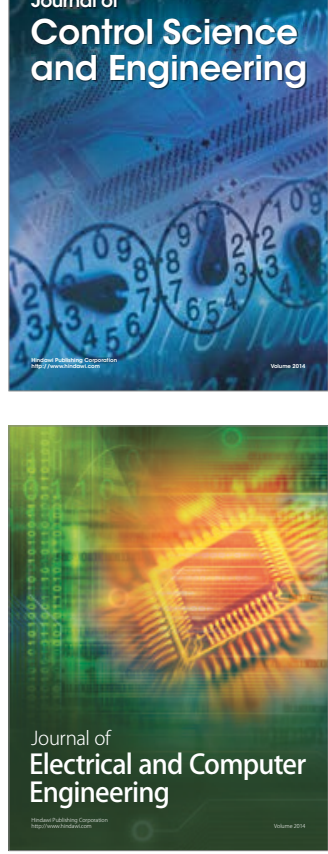

Distributed

Journal of

Control Science

and Engineering
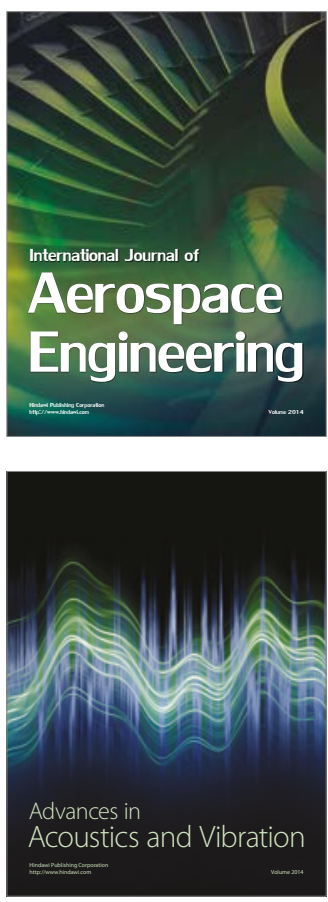

Sensor Networks 